\title{
Accession to Armament the Ambiguous Image Data and Authentication of Dispenser using Image Fusion
}

\author{
Abhijit G. Kalbande ${ }^{1}$ \\ Assistant Professor, Department of Electronic \& Telecommunication, P.R.M.C.E.A.M, Badnera, India ${ }^{1}$
}

\begin{abstract}
Image is collection of number of pixel, this pixel are nothing but the binary information which is either high or low $\mathrm{i}$; e (one or zero). Whereas fusion is process of joining two or more things together to form a single entity, due to which image fusion is one of technique that collect complete detail from different image. There in the fusion technique resultant fused image is very much useful for different task. This fusion image contain basic information which is important for human and machine perception by using wavelet transform we can obtain original distortion less secret image. There in this paper for getting distortion less image we used current wavelet transform because this edges in curvelet transform represent of edge is better that wavelet transform. In this paper contain implementation of image fusion using wavelet transform and curvelet transform.
\end{abstract}

Keywords: Fusion, Wavelet Family, Wavelet Transform, Wavelet base fusion, fused image, Curvelet Transform, Fast Fourier Transform, Inverse Fast Fourier Transforms and Ridgelet Transform.

\section{INTRODUCTION}

Image fusion is a task of linking the relevant information from a bunch of images into a single image, where the resultant fused image will be more informational and complete than any of the input images. Image fusion techniques can pick up the quality and enhance the uses of these data. Image fusion discover applications in the area of navigation intelligent, object finding and recognition, satellite imaging for remote sensing and civilian etc. Number of images required to be processed to obtain the clear information about the problem a patient has in the condition of medical territory, to fetch the information about the rebels or terrorists in the tract of military, to obtain the information about the fields which provide nice crop or mining spontaneity etc. In the field of geography, all these bid use the different kinds of images taken from various sources and processed based on the requirements by using various image processing gear.

The outcome of image fusion is a new image that gives the most beneficial information and physical appearance of each input image. It has been found that the standard fusion methods bring about well spatially but normally imported spectral distortion. To run over this difficulty numerous multi-scale transform based fusion schemes have been projected. Image fusion algorithms can be classified into different levels: pixel, feature and decision levels. Pixel level fusion operates directly on the pixels of source images while feature level fusion algorithms work on features extracted from the source images. Fig.1 show generalized diagram for image fusion. There is some important requisite for the image fusion process: The fused image should sanctuary all relevant information from the input images and the image fusion should not introduce artifacts which can lead to a wrong diagnosis. The fusion performance is calculated on the basis of the root mean square error (RMSE) and peak signal to noise ratio (PSNR).

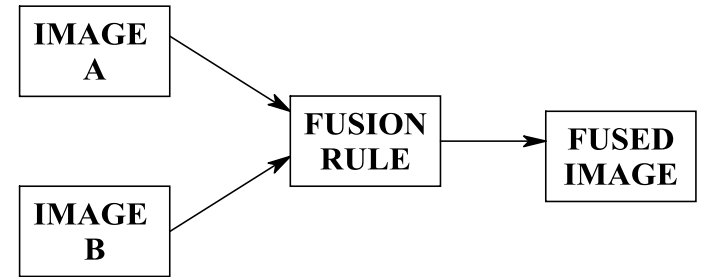

Fig.1:- Generalized block diagram for image fusion

\section{LITERITURE REVIEW}

In this paper, I am tendering relative analysis of image fusion using wavelet transform with different wavelet family and curvelet transform, also implementation of fusion using wavelet transform from different wavelet family and curvelet transform. certain associated works are mention here: P. Devaki, Dr. G. Raghavendra Rao,[1] suggesting an algorithm for protecting the secret image whose confidentiality needs to be sustained and also to validate the distributor who distributes that secret image to number of users. The secret image will be fused with the fingerprint of the dealer for authentication function. Fusion of the finger print will be done by using image fusion technique to create a single image consisting of the not only secret image but also the finger print image of the dealer. The fused image will be divided in two number of shares based on the threshold secret sharing technique. This provides both confidentiality of the secret image and as well as the certification of the dealer who has sent the image. The verification will be completed during reconstruction of the secret image. The secret image can be of medical, organizational information, military or any other sensitive information.

Deepak Kumar Sahu, M.P. Parsai,[2] granted a literature review on some of the image fusion techniques for image fusion like primitive fusion, discrete wavelet 
transform based fusion, Principal component analysis (PCA) based fusion etc. At last this review concludes that the image fusion algorithm based on combination of DWT and PCA with morphological processing will enhance the image fusion quality and may be the future trend of investigation regarding image fusion.

Myungjin Choi, Rae Young Kim, Moon-Gyu Kim,[3] analyze a new method based on the curvelet transform which represents edges better than wavelets. Since edges smash a fundamental function in image understanding, one good way to increase spatial resolution is to enhance the edges. Curvelet-based image fusion method provides good information in the spatial and spectral domains at a time. We realized IKONOS image fusion. This new method has reached an optimum fusion result.

Sweta Mehta, Prof. Bijith Marakarkandy Hari Om Shankar Mishra and Smriti Bhatnagar, [4] this paper introduces the curvelet transform and uses it to fuse images. In this paper we put forward an image fusion algorithm based on wavelet transform and the curvelet transform. The low and high frequency coefficients are selected according to different frequency domain after wavelet and the curvelet transform. In selecting the low frequency coefficients, the concept of local area variance was chosen to measuring criteria. In choosing the high frequency coefficients, the window property and local characteristics of pixels were reviewed. Finally, the projected algorithm in this article was applied to experiments of multi-focus image fusion and complementary image fusion.

Jean-Luc Starck, Fionn Murtagh, Emmanuel J. Candès, and David L. Donoho [5] contempt a new approach for contrast enhancement based on the curvelet transform. The curvelet transform represents edges superior than wavelets, and it is therefore suitable for the multiscale edge improvement. In a range of examples, use edge detection and segmentation, among other processing applications, to provide for quantitative comparative evaluation. Our findings are that curvelet based enhancement out-performs other enhancement methods on noisy images, but on near noiseless images curvelet based enhancement is not remarkably better than wavelet based enhancement.

\section{III.PROPOSED WORK}

In this work we have furnished the basics of wavelet transform, curvelet transform, implement image fusion using wavelet transform from various wavelet breeds and compare images obtained from wavelet families with curvelet transform used in image fusion. These outcomes are also distinguished with the processing of different images.

\section{A. Wavelet Transform}

Wavelet means "small wave" so wavelet analysis is about reviewing signal with short duration finite energy functions. Image fusions techniques are broadly differentiate in to two types. One is spatial domain fusion and the other one is transform based fusion. Spatial domain fusion can be performed based on averaging method, discrete wavelet transform based, principal component analysis based etc. Transform based fusion can be based on Fourier transform, wavelet transform etc. Wavelet transforms are multi-resolution image decomposition tool that provide a variety of channels representing the image feature by different frequency sub bands at multi-scale. It is a well know technique in analyzing signals. When decomposition is performed the approximation and detail component can be separated 2D discrete wavelet transformation converts the image from the spatial domain to frequency domain. The basic idea of wavelet transform is to represent any function $f$ as a linear superposition of wavelets. Any such superposition disintegrate $f$ to different scale levels, where each level can be then further decomposed with a resolution adapted to that level. One regular way to do this is writing $f$ as the sum of wavelets $\Psi \mathrm{m}, \mathrm{n}(t)$ over $m$ and $n$. This leads to discrete wavelet transform.

$$
\mathrm{f}(\mathrm{t})=\sum C m, n \Psi m, n(\mathrm{t})
$$

In wavelet transform, we use wavelets as transform basis. Wavelet functions are functions produced from one single function $\Psi$ by scaling and translation:

$$
\Psi a, b(\mathrm{t})=1 / \sqrt{ } a \Psi(t-b / a)
$$

The mother wavelet $\Psi(\mathrm{t})$ has to be zero integral, $\int \Psi(\mathrm{t}) \mathrm{dt}$ $=0$, High frequency wavelet correspond to $a<1$ or narrow width while low frequency wavelets correspond to a> 1 or wider width. Wavelets are functions defined over a finite interval and having an average value of zero. The basic idea of the wavelet transform is to represent any arbitrary function $\mathrm{f}(\mathrm{x})$ as a superposition of a set of such wavelets or basis functions. These basis functions are obtained from a single prototype wavelet called the mother wavelet $\Psi(\mathrm{x})$, by dilations or scaling and translations. Generalized block diagram for wavelet transform shown in fig.2. Wavelet base are very good at efficiently representing functions that are smooth except for a small set of discontinuities.

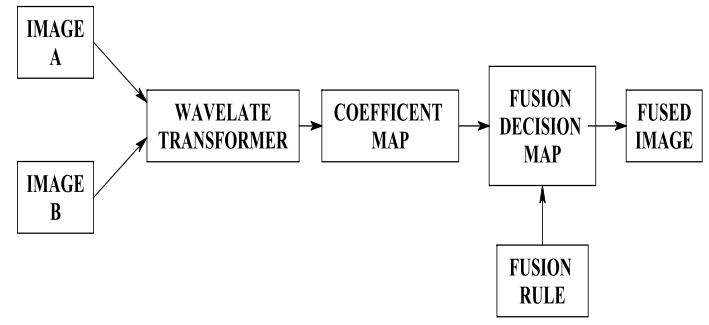

Fig.2:- Generalized block diagram for wavelet transform

In our experiment three types of wavelet families are examined: haar, daubechies, and biorthogonal.

- Haar: The first and simplest. Haar wavelet is discontinuous and resembles a step function. It represents the same wavelet as daubechies $\mathrm{db} 1$.

- Daubechies $(d b N)$ : The dbN wavelets are the daubechies external phase wavelets. $\mathrm{N}$ refers to the number of vanishing moments. Daubechies wavelets are families of orthogonal wavelets that are compactly supported. Compactly supported 
wavelets correspond to finite impulse response filters and thus lead to efficient implementations, thus making discrete wavelet analysis practicable.

- Biorthogonal: Biorthogonal exhibits the property of linear phase which is needed for signal and image reconstruction. By using two wavelets, one for decomposition (on the left side) and the other for reconstruction (on the right side) instead of the same single one interesting properties are derived

\section{B. Curvelet transform}

In this segment we audit the theory of ridgelet and curvelet transforms, the curvelet transform is a very young signal analyzing method with good potential. It is recognized as a milestone on image processing. The curvelet transform which represents edges better than wavelets. Curvelet transform is a tool for representation of curved shapes in images. Generalized block diagram of curvelet transform shown in fig.3. The idea is based on the segmentation of the complete image into small overlapping tiles and then applying ridgelet transform on each tile.

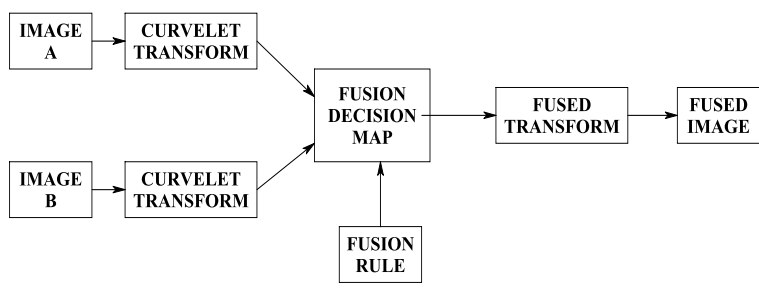

Fig.3.Generalised block diagram of curvelet transform

It is most approximate to work with medical images. Due to the limited capability of the wavelet transform to deal with images having curved shapes, the application of the curvelet transform for MR and CT image fusion is presented. MRI image provides much greater contrast between several soft tissues of body than CT image. Brightness of bones is higher in CT images but soft tissues can't be seen.

\section{IV.RESULT AND DISCUSSION}

The general necessity of an image fusing process is to preserve all valid and useful information from the source images, while at the same time it should not introduce any distortion in resultant fused image. Performance measures are used essential to measure the possible benefits of fusion. Image quality is measured using (PSNR). In this paper we calculated two PSNR and MSE terms.

A. Peak Signal to Noise Ratio (PSNR): It is the ratio between the maximum possible power of a signal and the power of corrupting noise that affects the fidelity of its representation. The PSNR measure is given by:

PSNR $(\mathrm{db})=20 * \log 10(255 / \mathrm{MSE})$
B. Mean Squared Error (MSE): The most commonly used error projection method where, the error value is the value difference between the actual data and the resultant data. The mean of the square of this error provides the error or the actual difference between the expected results to the obtained result. The difference between the pixel density of the perfect image and the fused image is squared and the mean of the same is the considered error. MSE value will be 0 if both the images are identical. The mathematical equation of MSE is given by the equation:

$\mathrm{MSE}=$ mse term $/(\mathrm{r} * \mathrm{c})$

In this work we have provided the basics of wavelet transform and after comparisons of different wavelets families and curvelet transform used in image fusion. These results are also compared for the processing of different images. The results of image fusion using wavelet transform from particular wavelet families are shown in Fig. (4), Fig. 4(a) Right plane Images (1) and (2) out of focus, respectively; (3) the resulting fused image using wavelet transforms shows two plane in focus. Fig .4(b) Left clock images (1) and (2) out of focus, respectively; (3) The resulting fused image using curvelet transforms shows two plane in focus.

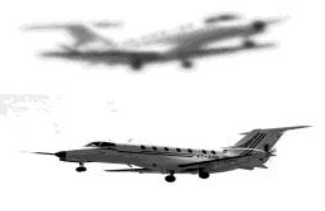

Image(1)
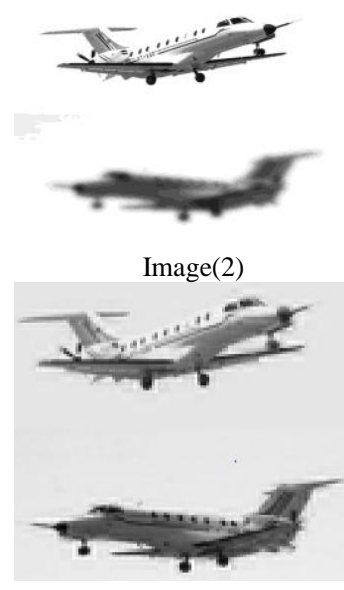

Image(3)

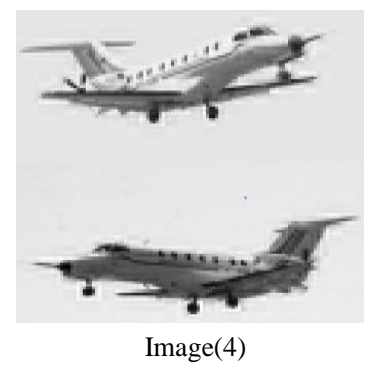

FIG.4 (a). PLANE

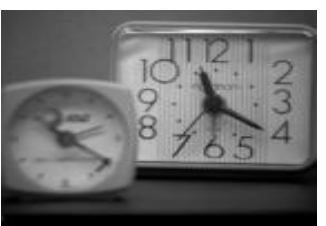

Image(1)
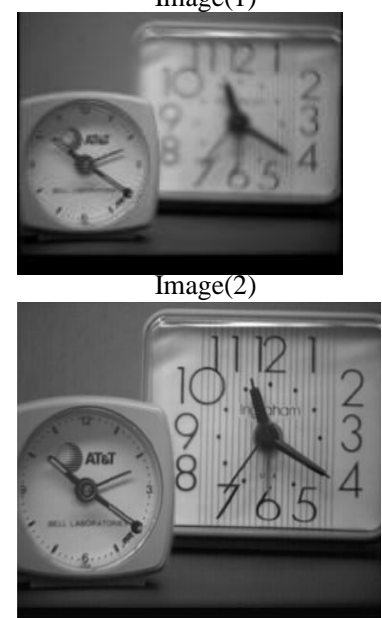

Image(3)

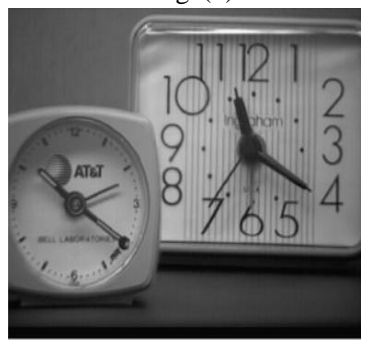

Image(4)

FIG.4.(b).CLOCK
Fig.4. Image fusion using wavelet transform and curvelet transform

For the above mentioned method their performance is measured in terms of MSE, PSNR and the results are 
tabulated in Table 1 for wavelet transform, Table 2 for curvelet transform and Table 3. Shows comparison of curvelet transform and wavelet transform.

TABLE. I.

COMPARISION OF WAVELET FAMILIES

\begin{tabular}{|l|l|l|l|c|c|c|c|}
\hline \multirow{2}{*}{$\begin{array}{l}\text { Sr } \\
\text { No }\end{array}$} & \multirow{2}{*}{\begin{tabular}{l} 
Name \\
\cline { 3 - 8 }
\end{tabular}} & & \multicolumn{4}{|c|}{$\begin{array}{c}\text { Waubechies } \\
\text { (for db3) }\end{array}$} & \multicolumn{2}{|c|}{ Haar } & \multicolumn{2}{c|}{$\begin{array}{c}\text { Bior } \\
\text { (Bior=1.5) }\end{array}$} \\
\cline { 3 - 8 } & & PSNR & MSE & PSNR & MSE & PSNR & MSE \\
\hline 1. & Plane & 5.945 & 128.6 & 5.668 & 132.7 & 5.55 & 134.5 \\
\hline 2. & Clock & 7.466 & 107.9 & 7.106 & 112.5 & 6.57 & 119.6 \\
\hline
\end{tabular}

TABLE. II

COMPARISION OF CURVELET FAMILIES

\begin{tabular}{|c|c|c|c|}
\hline \multirow{2}{*}{$\begin{array}{c}\text { Sr. } \\
\text { No. }\end{array}$} & \multirow{2}{*}{ Image } & \multicolumn{2}{|c|}{ Curvelet Transform } \\
\cline { 3 - 4 } & Name & PSNR & MSE \\
\hline 1 & Plane & 5.3242 & 138.14 \\
\hline 2 & Clock & 8.39 & 97.033 \\
\hline
\end{tabular}

TABLE .III

COMPARISION OF WAVELET TRANSFORM \& WAVELET TRANSFORM

\begin{tabular}{|c|c|c|c|c|c|}
\hline \multirow{2}{*}{$\begin{array}{c}\text { Sr } \\
\text { No }\end{array}$} & \multirow{2}{*}{ Image } & \multicolumn{4}{|c|}{ Fusion Method } \\
\cline { 3 - 6 } & & \multicolumn{2}{|c|}{$\begin{array}{c}\text { Wavelet } \\
\text { Transform }\end{array}$} & Curvelet Transform \\
\cline { 3 - 6 } & & PSNR & MSE & PSNR & MSE \\
\hline 1 & Plane & 5.941 & 128.66 & 5324 & 138.143 \\
\hline 2 & Clock & 7.466 & 107.9 & 83923 & 97.033 \\
\hline
\end{tabular}

\section{CONCLUSION}

It is probable to carry out image fusion using wavelet transform and curvelet transform has made great in the last four years so that choosing the wavelet, it is more crucial, successful to avoid highly complex and lengthy level decomposition. The proposed method provides a good result, both visually and quantitatively. The wavelet transforms is the very good technique for the image fusion gives a high quality spectral content. In this study, our results have shown that the wavelet transform is the valuable and analyses feature of images better.

\section{FUTURE SCOPE}

Future work performed does grip scope for further progress as a lot of research is happening in the field. Multi-wavelets based image fusion can be over and done to achieve a better image fusion quality. The efficiency of multi wavelets over the usual DWT methods in fusing images concern in remote sensing. The same can be applied in this project too and can be confirmed based on the image quality metrics developed. The image fusion value has been assessed based on optical image sets with respect to a perfect image. The productivity of the fusion can be better assessed if the same could be performed on many more multivariate images. The same could not be done due to lack of such set of test sample multivariate images.

\section{ACKNOWLEDGMENT}

First of all i am very much thankful to Myungjin Choi,Rae Young Kim, Myeong Ryong NAM, and Hong Oh Kim, who give the complete difference between wavelet transform and curvelet transform. Also I am very much thankful to Shrivsubramani Krishnamurthy, K.P. Soman who introduce the new fusion techniques, also i am very much thankful to all of them who directly or indirectly helping me for this research.

\section{REFERENCES}

[1] Myungjin Choi,Rae Young Kim, Myeong Ryong NAM, and Hong Oh Kim, "The Curvelet Transform for Image Fusion", International Journal of Engineering Science and Technology, 2000.

[2] Shrivsubramani Krishnamurthy, K.P. Soman, "Implementation and Comparative Study of Image Fusion Algorithms". International Journal of Computer Applications (0975- 8887) Volume 9- No.2, November 2010

[3] Digital Image Processing with MATLAB Gonzales and Woods $3^{\text {rd }}$ Edition.

[4] Sweta Mehta, Prof. Bijith Marakarkandy, "CT and MRI Image Fusion using Curvelet Transform", in journal of information, knowledge and research in electronics and communication engineering, 2013.

[5] Gonzalo Pajares, Jesus Manuel dela Cruz "A wavelet based image fusion tutorial" 2004 Pattern Recognition Society.

[6] http://www.mathworks.in/products/wavelet/examples.html?file=/pr oducts/demos/shipping/wavelet/imagefusiondemo.html.

[7] Susmitha Vekkots and Pancham Shukla a Novel Architecture for Wavelet based Image Fusion". World Academy of Science, Engineering and Technology, 2009.

[8] Sruthy S, Dr. Latha Parameswaran Ajeesh P. Sasi, "Image Fusion Technique Using DT-CWT" IEEE 978-1-4673-5090-7/13/2013.

[9] (2002) The IEEE website. [Online]. Available: http://www.ieee.org/

[10] Deepak Kumar Sahu, M. P. Parsai, "Different Image Fusion Techniques A Critical Review", International Journal of Modern Engineering Research (IJMER), Vol. 2, Issue 5, September 2012.FLEXChip Signal Processor (MC68175/D), Motorola, 1996.

\section{BIOGRAPHY}

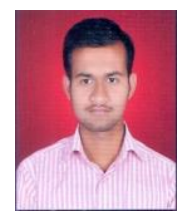

Prof. A G Kalbande is Assistant Professor in Electronic \& Telecommunication of Prof. Ram Meghe college of Engineering \& Management, Badnera-Amravati. He obtained his B.E \& M.E. (Electronic \& Telecommunication Engg) from Amravati university in 2013. His research interest "Digital Image Processing, VLSI \& Embedded". 